\title{
Telemonitoring and home hospitalization in patients with chronic obstructive pulmonary disease: study TELEPOC
}

Manuel Mirón Rubioa, Rocío Ceballos Fernández ${ }^{\mathrm{b}}$, Inmaculada Parras Pastorc, Amaya Palomo lloroa, 5 Borja Manuel Fernández Félix ${ }^{d}$, Jenifer Medina Miralles ${ }^{a}$, Esther Zamudio Lópeza , Javier González Pastora, Caridad Amador Lorente ${ }^{a}$, Nazaret Mena Hortelano ${ }^{a}$, Alejandro Domínguez Sánchez ${ }^{a}$ and Soledad Alonso-Viteri ${ }^{\mathrm{e}}$

aHome Hospitalization Unit, Torrejón University Hospital, Madrid, Spain; ${ }^{\circ}$ Nurse and healthcare manager, Torrejón University Hospital, Madrid, Spain; 'Cystelcom Sistemas S.A., Madrid, Spain; ${ }^{\text {IF } F a c u l t y ~ o f ~ S t a t i s t i c s, ~ C o m p l u t e n s e ~ U n i v e r s i t y, ~ M a d r i d, ~ S p a i n ; ~ ' N e u m o l o g y ~ D e p a r t m e n t, ~ T o r r e j o ́ n ~}$ University Hospital, Madrid, Spain

ABSTRACT

Background: Chronic obstructive pulmonary disease (COPD) is a major consumer of healthcare resources, with most costs related to disease exacerbations. Telemonitoring of patients with COPD may help to reduce the number of exacerbations and/or the related costs. On the other hand, home hospitalization is a cost-saving alternative to inpatient hospitalization associated with increased comfort for patients. The results are reported regarding using telemonitoring and home hospitalization for the management of patients with COPD.

Methods: Twenty-eight patients monitored their health parameters at home for six months. A nurse remotely revised the collected parameters and followed the patients as programmed. A home care unit was dispatched to the patients' home if an alarm signal was detected. The outcomes were compared to historical data from the same patients.

Results: The number of COPD exacerbations during the study period did not reduce but the number of hospital admissions decreased by $60 \%$ and the number of emergency room visits by $38 \%$. On average, costs related to utilization of healthcare resources were reduced by $€ 1,860.80$ per patient per year.

Conclusions: Telemonitoring of patients with COPD combined with home hospitalization may allow for a reduction in healthcare costs, although its usefulness in preventing exacerbations is still unclear.
ARTICLE HISTORY

Received 12 June 2017

Accepted 14 February 2018

\section{KEYWORDS}

COPD; hospital at home; home care units; home hospitalization telemedicine; telemonitoring

\section{Introduction}

Telemonitoring is a patient management approach that uses communication technology to monitor health status at distance. In the recent years, its use has increased dramatically, fueled by a rampant technological progress, and has gained a growing interest as a tool to remotely follow patients with chronic diseases. Among its potential benefits are improved patient-doctor communication, increased patient's autonomy, reduced hospitalization rate, lower costs for the healthcare system, easier control of patients living in remote areas, and possible early detection of disease exacerbations [1-4]. Not all the authors, however, find telemonitoring advantageous [5], and some even indicate that it may harm doctor-patient relationship or result in over-treatment [6].

Various schemes of telemonitoring have been described: multiple available methodologies and technologies can be used alone or in combination [1]. Telemonitoring using electronic devices can be combined with regular or on-demand phone interviews. In general, the patient monitors his/her health parameters at home using electronic devices, and the data are transmitted to the health care center. The data to be collected can vary from a symptom diary filled out on a touch screen to clinical parameters measured with electronic devices. The data can be transmitted automatically by the measuring/registering device itself or by the patient via phone or internet. In some models, the data are received and revised regularly by a nurse or a doctor who intervene if an alarm signal is detected. In other models, the data are collected via a call center, which detects abnormal values and informs the health care professional in charge. Whatever the model used, telemonitoring in itself only allows for timely detection of alarm signals, upon which an action is required. The action can vary from self-administration of rescue medication according to physician's indications to patient's visit to a hospital or an emergency room. Thus, telemonitoring alone cannot provide comprehensive health care and fully abolish the need for intervention by health care professionals or hospitalization.

Home hospitalization is another strategy that allows to reduce health care costs by shortening hospital stay and/or avoiding hospital admission [7]. In this care scheme, doctors/nurses travel to patients' home where they provide acute or subacute care. Home hospitalization helps to increase patients' physical and psychological comfort and reduce the risk of nosocomial infections while maintaining high standards of care. Nevertheless, the lack of constant proximity of and observation by the hospital staff may lead to a failure to detect early alarm signs and may result in an emergency room visit. 
Chronic obstructive pulmonary disease (COPD) represents a huge economic burden for the healthcare system worldwide. Telemonitoring has been proposed as means both to improve patient's clinical outcomes and quality of life and to allow for cost reduction. Results from several studies indicate that telecare could help to detect early signs of disease exacerbations and to reduce the need for hospitalization, thereby producing a saving for the health care system [2,3]. In the same way, some studies have shown that nurse-centered teleassistance allows for a reduction in hospital admissions [8,9], and visits to the emergency department [10]. On the other hand, some studies evaluated the outcomes of home hospitalization during COPD exacerbations $[11,12]$ and found that it was associated with higher comfort for the patients, savings for the healthcare system and similar clinical outcomes, compared to conventional inpatient hospitalization. To our knowledge, to date there have been no studies on combining telemonitoring and home hospitalization as a COPD management approach.

The objective of the present study was to evaluate the usefulness of a combined strategy based on telemonitoring and home hospitalization in reducing the number of hospital admissions and emergency room visits in patients with COPD.

\section{Patients and methods}

\subsection{Study design}

This was a single-arm prospective unicenter study that compared the results of applying the telemonitoring and home hospitalization strategy during the study period with the historical results of the same patients.

The study was carried out between February and September of 2013. All the patients were enrolled within 2 months and were followed for 6 months (study period).

105 The outcomes were compared to the data from the same months during the previous year, to minimize confounding due to seasonal variation. The data for the control period were collected retrospectively from patients' medical records.

The selection process was based on the revision of the medical records of patients diagnosed with COPD and personal interview. The sample size was estimated based on feasibility (28 monitoring systems were available).

\subsection{Human resources}

The home care unit (HCU) was composed of two physicians (an internist and a geriatrist), four nurses, and one nurse supervisor. During the week, the physicians carried out their usual work at the hospital between 8.00 and 15.30. On weekends and non-working days, the physicians were on calls between 8.00 and 21.00 . The standard working day of the nurses was from 8.00 till 21.00 on any day of the year. In case of emergency, the patients could call the hospital guard at night. The hospital reference population was 118,000 inhabitants, and the HCU could attend a maximum of 21 patients per day. For the purposes of the study, a specifically assigned nurse dedicated $8 \mathrm{~h}$ per week to data monitoring and patient follow-up. The nursing staff did not have a specific certified training in pneumology. However, there were nurses specifically devoted to respiratory diseases who could be consulted by the nurse in charge of monitoring in case of need. This latter nurse could also consult with the physician specialized in pneumology. The pulmonologist participated in patient selection and visited the study patients in outpatient clinic with the usual frequency. No other additional human resources were involved.

\subsection{Technological resources}

Electronic devices were used to measure heart rate, blood pressure, and peripheral oxygen saturation. The devices were connected to a communication hub via Bluetooth. The hub contained a SIM card and was continuously connected to an electricity source via a conventional wall plug. The data were automatically transmitted to the administration web platform where they could be consulted by the study team from any device with internet connection. When the obtained values were outside the normal range, the telemonitoring system generated an alarm signal that was sent to the smartphone of the nurse in charge of telemonitoring or, in case of her unavailability, to the study physicians; the values were also registered in the web platform. As a general rule, an alert was activated for all patients with an $\mathrm{O} 2$ saturation $<92 \%$ (regardless of the use of chronic oxygen therapy), heart rate $>100$ bpm or $<60 \mathrm{bpm}$, and systolic blood pressure $>140 \mathrm{mmHg}$, but normal ranges could be personalized to fit clinical characteristics of each patient. Patients were instructed to measure the parameters once a day and each time they presented respiratory symptoms. All technological support was provided by Cystelcom Sistemas S.A. (Madrid, Spain).

The participants received face-to-face instructions on correct use of the measuring devices. A study team nurse visited the patients in their home to confirm the correct assimilation of the instructions. In case of technical issues the patients could contact the provider of the electronic devices.

\subsection{Description of intervention}

Telemonitoring program consisted of two strategies: follow-up and control by a nurse during the stable phase and intervention by the $\mathrm{HCU}$ team during disease exacerbations. During the stable phase, the nurse in charge revised the data collected in the web platform every working day, carried out a structured phone interview with every patient once a week and visited the patients in their home once a month. At each scheduled visit, nurses assessed the inhalation technique and made recommendations on lifestyle. In addition, the nurse called the patients when an out-of-range value was received. The patients could also contact the nurse telephonically.

During visits or phone calls the nurse could make therapeutic recommendations, that is, on the use of bronchodilators, mucolytics, and respiratory physiotherapy. Patients who felt worse in the evening or in the night on a week day or during the weekend were instructed to go to the hospital emergency room where they were identified as the study participants, and their visits were registered. Patients were instructed to contact their primary care physician for any health problems unrelated to respiratory symptoms. 
When an out-of-range value was received in the web platform (or after a clinical worsening reported by the patient over the phone, even if no alarm was detected), the monitoring nurse administered a symptoms questionnaire over the phone (Figure 1). That means that oxygen saturation was evaluated as an additional parameter within the patient's clinical picture along with other signs and symptoms of COPD exacerbations (dyspnea, sputum volume and purulence, axillary temperature). Therefore, if an isolated and non-significant fall in oxygen saturation was detected, no specific recommendations were given, although a follow-up was implemented to detect the appearance of new symptoms. A COPD exacerbation was defined as a 'sustained worsening of the patient's condition, from the stable state and beyond normal day-to-day variations that is acute in onset and may warrant additional treatment in a patient with underlying COPD'.

Any COPD exacerbation detected through the questionnaire was reported by the nurse to the doctor (an internist or geriatrist) at the home hospitalization unit. Then, it was these doctors who made the decision to either refer the patient to her/his health center to be followed by her/his primary care physician; order daily check-ups by the nurse at the telemonitoring center; carry out a home-based assessment; create an episode of home hospitalization; or transfer the patient to the Emergency Department if symptoms were severe. Both the indications and the management of patients in hospital at home during the control period were the same as during the study period.

Exclusion criteria for admission in the HCU were: deterioration of consciousness, acute confusional state, new-onset cyanosis or severe dyspnea with use of accessory muscles, arterial $\mathrm{pH}<7.35$ on arterial blood gas analysis (if this was done), acute changes on chest- $X$ ray, acute EKG changes, if an EKG was performed, and concomitant medical disorders requiring hospitalization. No specific criteria were defined neither for referral of the patient to her/his primary care physician nor for the decision to refer the patient to the emergency department

\section{SYMPTOM QUESTIONNAIRE}

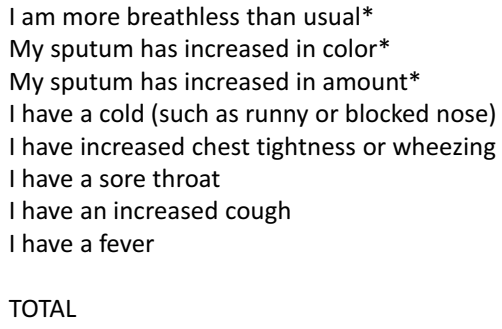

Figure 1. Symptom questionnaire and intervention protocol. that were not the impossibility of visiting the patient immediately in case of seriousness or that the patient themselves voluntarily expressed the desire to go to the emergency department.

Patients who were eventually hospitalized at home received standard care provided by the HCU. The measurements and data collection/transmission were identical during the acute and the stable phase, but during the acute phase the data were analyzed by the HCU personnel.

\subsection{Study population}

The inclusion criteria were: patients with spirometry-confirmed diagnosis of COPD; at least two hospital admissions or one hospital admission and two or more emergency room visits due to a COPD exacerbation during the 12 months prior to the inclusion in the study; residency in the geographic area covered by the $\mathrm{HCU}$; home condition adequate for carrying out clinical practice, and availability of a caregiver $24 \mathrm{~h}$ per day. The exclusion criteria were: diagnosis of asthma or another respiratory disease different from COPD; end-stage COPD; history of lung volume reduction surgery or lung transplant; tracheostomy; any serious comorbidity that could interfere with the study and cause life expectancy $<6$ months; significant functional dependency (Barthel index $[13,14]$ value $<15$ ); severe cognitive impairment (Short Portable Mental Status Questionnaire [15] score > 8); poor treatment adherence; patient's or caregiver's incapacity to use the study devices, and participation in a another clinical trial.

Withdrawal criteria were: serious adverse events; protocol deviations that could affect the validity of the results, and consent withdrawal.

\subsection{Study endpoints}

Primary endpoints were incidence and mean duration of hospital admissions and incidence of emergency room visits. In addition, the following data were collected and analyzed: demographic data (age, gender), clinical data (COPD severity, use of long-term oxygen therapy; body mass index); use of other healthcare resources (primary care visits; hospitalization in intensive care units); healthcare costs of acute episodes; patient-perceived quality of care and user's and technical evaluation of the telemonitoring system. At the beginning and at the end of the study period we collected data from Charlson comorbidity index [16]; performance in activities of daily living as measured by Barthel index; the modified Medical Research Council breathlessness scale score [17,18]; health-related quality of life as measured by COPD assessment test [19]; and the body mass index. Rates to calculate costs were taken from a publication by the Galician Health Service [20]. These figures were used because this was the only reference data available at the time the manuscript was written. The prices shown include all those costs likely to be incurred by a home hospitalization unit (personnel, drugs/medicines, transportation, etc.). A 10-item ad-hoc questionnaire, with each item rated as 'I agree' or 'I disagree', was developed for the evaluation of the patient-perceived quality of care. This 
questionnaire is not validated, although it is similar to those used in other studies. The questions were designed based on those aspects the investigators considered to be more relevant to analyze the results.

\subsection{Ethical conduct of the research}

The study was designed and carried out in agreement with the

280 Declaration of Helsinki. The study protocol was approved by the Ethics Committee of University Hospital of Torrejon. All the patients signed an informed consent prior to being included in the study.

\subsection{Statistical analysis}

285 No formal calculation of sample size was performed; the number of patients was defined based on feasibility. Non-parametrical tests were used for statistical analysis. The differences between the intervention and the control periods were assessed using Wilcoxon signed-rank test for continuous variables and McNemar test for categorical variables. Data from the two patients who died during the study were included in the analysis; for these patients, the calendar period from the previous year equivalent to the period of their participation (from enrolment to study termination) was used as control.

\subsection{Study funding}

Cystelcom Sistemas S.A. assumed the costs of the technology (devices, internet connection). Funds from the University Hospital of Torrejón were used to finance the services of the medical writer.

\section{Results}

\subsection{Patient disposition}

Twenty-eight patients were enrolled in the study. Twenty-six patients completed the 6-month telemonitoring period. Two patients died during the study: one died in the intensive care unit from a septic shock that originated from a communityacquired pneumonia, and the other died during a conventional hospitalization from respiratory and multiple organ failure secondary to a community-acquired pneumonia. Neither patient was being cared by the home hospitalization unit at the time of onset of respiratory symptoms.

\subsection{Baseline characteristics}

Most patients $(26 / 28)$ were males, and the mean age was 78 years (range 58-96 years; standard deviation [SD] $=7.9$ years). The mean baseline FEV1 was $50.8 \%$ (range

$31528-80 \%$; SD = 18.3). During the study two patients were active smokers. Baseline clinical and demographic characteristics are shown in Table 1.
Table 1. Patients' clinical and demographic characteristics.

\begin{tabular}{|c|c|c|c|}
\hline & Baseline $(n=28)$ & 6 months $(n=26)$ & $\begin{array}{c}p \\
\text { value }\end{array}$ \\
\hline Males, $n(\%)$ & $26(93)$ & & \\
\hline Age (years), mean (SD) & $78(7.9)$ & & \\
\hline \multicolumn{4}{|l|}{ COPD Severity, $n(\%)$} \\
\hline GOLD I & 1 (3.6) & & \\
\hline GOLD ॥ & $14(50)$ & & \\
\hline GOLD III & $10(35.7)$ & & \\
\hline GOLD IV & $3(10.7)$ & & \\
\hline LTOT, $n(\%)$ & $11(39)$ & & \\
\hline $\begin{array}{l}\text { Charlson comorbidity score, } \\
\text { mean (SD) }\end{array}$ & $3.4(1.4)$ & $4.4(1.8)$ & $<0.05$ \\
\hline Barthel index, mean (SD) & $78.5(22.8)$ & $73.8(31.9)$ & 0.02 \\
\hline Body mass index, mean (SD) & $28.6(5.6)$ & $28.3(5.1)$ & 0.5 \\
\hline $\begin{array}{l}\text { Modified breathlessness MRC } \\
\text { score, mean (SD) }\end{array}$ & $2.9(0.8)$ & $2.8(0.8)$ & 0.2 \\
\hline CAT score, mean (SD) & $21.6(4.9)$ & $20.7(5.2)$ & 0.08 \\
\hline
\end{tabular}

SD: standard deviation; CAT: chronic obstructive pulmonary disease assessment test; MRC: Medical Research Council; COPD: chronic obstructive pulmonary disease; GOLD: global initiative for chronic obstructive lung disease; LTOT: long-term oxygen therapy.

\subsection{Effectiveness of the intervention}

Clinical characteristics of the patients did not change significantly from baseline to end of study, except for Charlson comorbidity index, which raised from 3.4 points at baseline to 4.4 points at final visit $(p<0.05)$, and Barthel performance index, which diminished from 78.5 to 73.8 points $(p=0.02$ ).

A total of 72 exacerbation episodes were registered in a total of $25(89.3 \%)$ patients during the study period (mean: 2.6 episodes per patient), versus 59 exacerbations in 26 (92.9\%) patients during the control period (mean: 2.1 episodes per patient; $p=0.21$ ). It can be seen that the number of patients included in the study were 28 , however, only 26 had exacerbations during the control period. These findings can be explained because the inclusion of patients was based on the exacerbations (two hospital admissions or one admission plus two or more visits to the Emergency Department) occurring throughout a whole year (2012). However, the control period with which the study period was compared lasted only 6 months for each patient. In other words, some patients may have had exacerbations during year 2012 out of the control period, although these exacerbations could have been used as inclusion criteria for the study patients.

Activities of the telemonitoring nurse and the HCU performed during the study are summarized in Table 2. The most common actions performed by the telemonitoring nurse were programmed calls $(n=589)$, programmed visits to patients' homes $(n=159)$, and therapeutic recommendations, especially on physiotherapy $(n=236)$. These recommendations were made by the telemonitoring nurse whenever the patient experienced an increased sputum volume or dyspnea, but with no other symptoms of serious or infectious exacerbation, such as fever or increased sputum purulence. These recommendations were provided over the phone, and a daily follow-up was implemented to assess whether clinical manifestations remitted o worsened. The only physical therapy resource recommended was the use of an incentive spirometer to facilitate expectoration. Scheduled visits were performed as per calendar, and were independent of patients' 
Table 2. Actions performed by the telemonitoring nurse (autonomously or after consulting the HCU physicians) and by the HCU during the study period.

\begin{tabular}{|c|c|c|}
\hline \multicolumn{2}{|c|}{ Actions } & $N$ \\
\hline \multicolumn{3}{|c|}{ Actions performed by the telemonitoring nurse } \\
\hline \multirow[t]{5}{*}{ Visits and phone calls } & Programmed visits & 159 \\
\hline & Emergency visits & 20 \\
\hline & Programmed phone calls & 589 \\
\hline & Not programmed phone calls & 52 \\
\hline & Phone call received from patients & 77 \\
\hline \multirow[t]{4}{*}{ Referrals to } & Primary care & 24 \\
\hline & Pneumologist (outpatient office) & 5 \\
\hline & Other specialists (outpatient office) & 5 \\
\hline & COPD-related home hospitalization & 32 \\
\hline \multirow[t]{3}{*}{ Therapeutic recommendations } & Use of bronchodilators & 81 \\
\hline & Use of mucolytics & 51 \\
\hline & Physiotherapy & 236 \\
\hline \multicolumn{3}{|l|}{ Actions performed by the HCU } \\
\hline \multirow[t]{4}{*}{ Home hospitalization requested by } & Primary care & 1 \\
\hline & Emergency room & 12 \\
\hline & Hospital ward & 14 \\
\hline & Telemonitoring nurse & 32 \\
\hline
\end{tabular}

COPD: chronic obstructive pulmonary disease; HCU: home care unit.

clinical course. The most frequent referrals were to hospital at home $(n=32)$. Not all patients with exacerbation of COPD admitted to home hospitalization did so at the request of the nurse in charge of telemonitoring. Some were referred from the Emergency Department and others had previously gone through conventional hospitalization. That is why not all home hospitalization admissions during the study period $(n=57)$ were performed at the request of the monitoring nurse.

Utilization of healthcare resources during the study and the control periods is summarized in Table 3 . In relation to EPOC, healthcare resources were used on 110 occasions during the study period and on 110 occasions during the control period. Thirteen conventional hospital admissions occurred during the study period (mean: 0.46 admissions per patient; range 0-4), compared with 33 hospital admissions during the control period (mean: 1.18 admission per patient; range 0-4; $p=0.0067$ vs study period), which means a reduction of $60 \%$ during the intervention period. The number of admitted patients was $21(75.0 \%)$ during the control period and 7 (25.0\%) during the study period. There was a $58 \%$ reduction in the total number of days spent in the hospital, from 212 days during the control period to 89 days during the study period. The average length of conventional hospitalization was similar during both periods: 6.84 days (range: $3-10$ ) during the study period, and 6.42 days (range: $2-39$ ) during the control period ( $p=0.82$ ). The number of visits to emergency room decreased by 38\%, from 53 visits during the control period (in 26 (92.9\%) patients; mean 1.89 visits/patient; range $0-6$ ) to 33 visits during the telemonitoring period (in 15 (53.6\%) patients; mean 1.18 visits/patient; range $0-6, p=0.03$ ).

In general, fewer patients had to be admitted to the hospital or to go to emergency room during the telemonitoring period: only 15 patients $(53.6 \%)$ had to stay in hospital or present in the emergency room during the study period, compared to 26 (92.8\%) patients during the control period (RR 0,58; IC 95\% 0,40 - 0,83 $p=0.002$ ). Such reduction translated into higher number of home hospitalizations, which occurred on 57 occasions during the study period (in 23 (82.1\%) patients; mean: 2.04 home hospitalizations per patient, range: $0-7)$ versus 12 occasions during the control period (in 8 (28.6\%) patients; mean: 0.4 home hospitalizations per patient, range: $0-4 ; p<0.001)$. The average duration of home hospitalizations was similar between the two periods (9.6 days, range: $0-26$ days during the study period, and 11.4 days, range: 5-22 days during the control period, $p=0.31)$.

\subsection{Costs of utilization of healthcare resources during control and telemonitoring periods}

We analyzed the costs of utilization of healthcare resources during the control and the telemonitoring periods, using reference prices for each type of resource (Table 4). Reference costs of bed per day were used for calculating the total costs of inpatient hospitalization, home hospitalization and ICU stays. Overall, telemonitoring combined with home hospitalization was associated with reduced costs related healthcare resource utilization (an estimated total of $116,274.50 €$ during the study period versus $142,325.8 €$ during the control period).

\subsection{Patient-perceived quality of care and technology acceptance}

Thirteen patients ( 11 males and 2 females, mean age 75.4 years, range 58-84) completed the ad-hoc satisfaction questionnaire. The results are shown in Figure 2. The degree of satisfaction was high (at least $77 \%$ ) for all items covered by the questionnaire. The patients were also asked how much time they had to invest into learning how to use the technology. Six (46\%) patients

Table 3. Utilization of healthcare resources during the study and the control periods.

\begin{tabular}{|c|c|c|c|}
\hline Type of healthcare resource & $\begin{array}{l}\text { Study period } \\
\quad(n=28)\end{array}$ & $\begin{array}{c}\text { Control period } \\
(n=28)\end{array}$ & $p$ \\
\hline Hospital admission, $n$ & 13 & 33 & \\
\hline Emergency room visit, $n$ & 33 & 53 & \\
\hline Home hospitalization, $n$ & 57 & 12 & \\
\hline Primary care visit, $n$ & 5 & 12 & \\
\hline Admission to intensive care unit, $n$ & 2 & 0 & \\
\hline Total & 110 & 110 & \\
\hline Hospital admission episodes per patient, mean (range) & $0.46(0-4)$ & $1.18(0-4)$ & 0.006 \\
\hline Emergency room visit per patient, mean (range) & $1.18(0-6)$ & $1.89(0-6)$ & 0.03 \\
\hline Home hospitalization episodes per patient, mean (range) & $2.04(0-7)$ & $0.4(0-4)$ & $<0.001$ \\
\hline Primary care visit per patient, mean (range) & $0.18(0-1)$ & $0.43(0-3)$ & 0.114 \\
\hline Hospital LOS mean (range) & $6.84(3-10)$ & $6.42(2-39)$ & 0.82 \\
\hline Home hospitalization LOS mean (range) & $9.6(0-26)$ & $11.4(5-22)$ & 0.31 \\
\hline
\end{tabular}

LOS: length of stay; SD: standard deviation. 
Table 4. COPD-related costs of healthcare resources utilized during the study and the control periods.

\begin{tabular}{|c|c|c|c|c|c|}
\hline Type of healthcare resource & $\begin{array}{c}\text { Times of resource use, } n \\
\left(\%^{\mathrm{a}}\right)\end{array}$ & $\begin{array}{l}\text { Total number of } \\
\text { days }\end{array}$ & Cost/day & $\begin{array}{l}\text { Mean cost/ } \\
\text { event }\end{array}$ & $\begin{array}{l}\text { Total costs per type of } \\
\text { resource }\end{array}$ \\
\hline \multicolumn{6}{|l|}{ Study period $(n=28)$} \\
\hline Hospital admission & $13(25.0)$ & 89 & $526.00 €$ & $3601,08 €$ & $46,814.00 €$ \\
\hline Emergency room visit & $33(53.6)$ & - & - & $359.00 €$ & $11,847.00 €$ \\
\hline Home hospitalization & $57(82.1)$ & 547 & $80.00 €$ & $767.71 €$ & $43,760.00 €$ \\
\hline Primary care visit & $5(17.9)$ & - & - & $68.90 €$ & $344.50 €$ \\
\hline Admission to intensive care unit & $2(7.1)$ & 7 & $1,136.00 €$ & $3,976.00 €$ & $7,952.00 €$ \\
\hline $\begin{array}{l}\text { Telemonitoring nurse (8h/week during } \\
32 \text { weeks) }\end{array}$ & & & & & $5.557,00 €$ \\
\hline \multicolumn{6}{|l|}{$\begin{array}{l}\text { Total costs during study period } \\
116,274.50 €\end{array}$} \\
\hline \multicolumn{6}{|l|}{ Control period $(n=28)$} \\
\hline Hospital admission & $33(75.0)$ & 212 & $526.00 €$ & $3,379.15 €$ & $111,512.00 €$ \\
\hline Emergency room visit & $53(92.7)$ & & - & $359.00 €$ & $19,027.00 €$ \\
\hline Home hospitalization & $12(28.6)$ & 137 & $80.00 €$ & $913.33 €$ & $10,960.00 €$ \\
\hline Primary care visit & $12(32.4)$ & & - & $68.90 €$ & $826.80 €$ \\
\hline Admission to intensive care unit & 0 & 0 & $1,136.00 €$ & $0 €$ & $0 €$ \\
\hline $\begin{array}{l}\text { Total costs during control period } \\
142,325.8 €\end{array}$ & & & & & \\
\hline
\end{tabular}

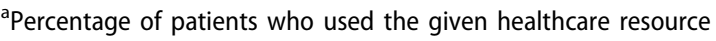

invested one day or less, four (31\%) patients invested more than one day but less than one week, and three (23\%) patients invested between one and two weeks. We did not evaluate the caregiver burden, although we did not receive any complaint during the study period regarding frequency of assessments (phone calls and scheduled visits) or the recording of clinical parameters (at least once daily).

\subsection{Technical evaluation of the telemonitoring system}

430 Twenty-eight technical incidents were registered during the study. The most frequent event $(n=14)$ was an erroneous perception by the patient that the device was malfunctioning Other incidents included suboptimal internet connection ( $n=5)$, malfunctioning of communication devices $(n=4)$, battery replacement $(n=4)$, and device breakage $(n=1)$.

\section{Discussion}

The results of the present non-randomized single-arm interventional study demonstrate that using telemonitoring and home hospitalization for management of patients with COPD may reduce the number of hospital admissions and emergency room visits and to diminish the costs related to healthcare resource utilization.

In theory, close monitoring of clinical parameters might allow for early detection of symptom deterioration and timely intervention, thus preventing a fully blown exacerbation. Indeed, several randomized controlled studies indicate that this may be the case $[21,22]$. In our study, however, the studied approach did not lead to reduction in the number of exacerbations. Several reasons may contribute to this inconsistence. First, use of historical control could be associated with data loss and underestimation of real number of exacerbation during the control period. Second, COPD is a progressive disease, and most participants were seriously ill, of advanced age and with a significant comorbidity burden; their health may have declined during the months separating the control and the study period. Finally, this inconsistence could be due to differences in the telemonitoring approach and the standard care across the studies.

In our study, the combined strategy of telemonitoring and home hospitalization in patients with COPD helped to reduce

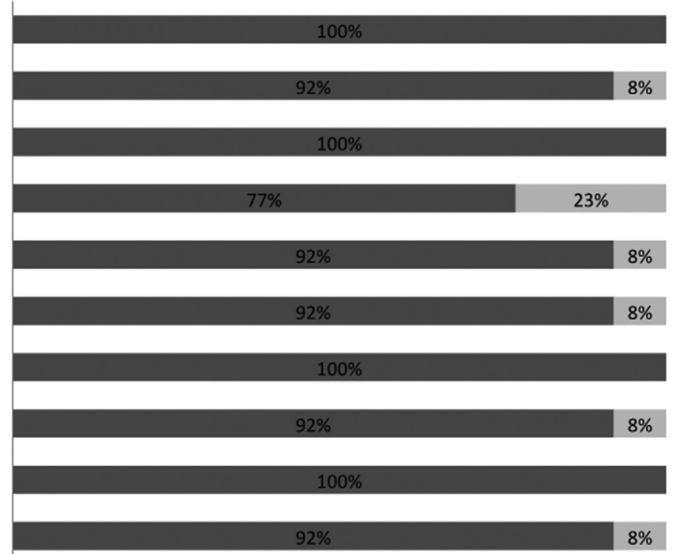

Figure 2. Patient-perceived quality of care and technology acceptance. 
the number of conventional inpatient hospitalizations by $60 \%$, compared to the control period in the previous year in the same group of patients. Several previous studies focusing on telemonitoring alone also reported a reduction in the number of hospital admission in patients with COPD followed via telemonitoring, but the effect was smaller. Thus, in a recent randomized controlled trial, Pedone et al. [22] found that the number of hospital admissions in the telemonitoring group was 33\% lower than in the control group that received standard care. Other randomized studies reported a reduction of $32 \%-46 \%$ in the number of hospital admissions $[21,23]$. It seems that in our case the combination of telemonitoring with home care unit implication permitted to treat most exacerbations in the patients' home, without the need for inpatient hospitalization.

It should be noted that during the study, the nurse in charge of the monitoring performed the control of the patients only on weekdays and during the day. In case of symptom worsening on weekends, holidays and at night, patients were instructed to contact the emergency services. Therefore, we believe that providing telemonitoring services during the night and on weekends may have allowed to avoid a few further hospital admissions and emergency department visits during the study period.

When the hospitalization did occur during the study period, the length of hospital stay was similar to the one during the control period. In contrast, some other studies reported shortening of hospital stay associated with telemonitoring [24]: thus, in the randomized controlled study by Bourbeau et al. [21], the average length of hospital stay was shorter in the group receiving telephonic support, compared to the standard care group (7.2 \pm 19.5 versus $12.5 \pm 21.2$ days), similar to the study by Lewis et al. ( $7.43 \pm 15.6$ versus $18.2 \pm 24.55$ days). However, other studies did not find shortening of hospital stay in telemonitoring groups [2] or even showed the opposite effect [25].

In agreement with previous reports [23], the number of emergency room visits in our study was reduced by $38 \%$, compared to the control period.

In a recent observational, controlled study, the intervention group (telemonitoring and education provision on COPD) showed a reduction, as compared to the usual care group, in the number of hospital admissions (OR $0.3895 \% \mathrm{Cl}$ $0.27-0.54 ; p<0.0001$ ), emergency department visits (OR $0.5695 \% \mathrm{Cl} 0.35-0.92 ; p<0.02)$, readmissions within 30 days of discharge (OR $0.4695 \% \mathrm{Cl} 0.29-0.74 ; p<0.001$ ), and cumulative length of hospital stay (OR $0.5895 \% \mathrm{Cl} 0.46-$ 0.73; $p<0.0001$ ) over a two-year follow-up period [26]. The reduction rates of admissions and emergency department visits are similar to those observed in our study, although duration of follow-up was 6 months in our study, the period during which the technology-supplying company provided us with both the devices and the platform for telemonitoring. Longer follow-up periods would be necessary to check if the reduction in the number of admissions and emergency department visits is maintained.

As expected, fewer visits to emergency room and inpatient hospitalizations translated into a higher number of home hospitalizations ( 57 home hospitalizations during study period versus 12 during the control period). Moreover, the average length of home hospitalization during the study was similar to the control period. We do not have a definite explanation for this finding. It might well be that, although remote monitoring did allow for a better knowledge of a patient's status at any moment, it did not result in a shortening of treatment periods. We do not know if, because of disease progression, the exacerbations observed during the interval between the control period and the study period were more serious and required more days of control and follow-up. Therefore, this is an aspect that we should consider for future studies.

The Charlson index increased 1 point in only six months. It might be due to some patients being diagnosed of highly prevalent conditions, such as heart failure, cerebrovascular disease, diabetes mellitus, or diabetic end-organ damage (retinopathy, nephropathy, neuropathy) during the study period. Similarly, there was a significant reduction in Barthel's index, which could be explained by a COPD progression and/or increased comorbidity.

In our study, costs related to utilization of healthcare resources were reduced, compared to the control period, producing a saving of $26,051.3 €$ for 28 patients over 6 months. This suggests that the described program could produce an average saving of $1,860.8 €$ per patient per year. Due to the devices being given free of charge to the institution where the study was done, this calculation does not take into account the costs of device purchase and monitoring system installation (capital investment) and costs of technical maintenance, which can vary substantially. A different result would have been obtained if these costs had been included; it should be mentioned, however, that costs of technology are continuously decreasing, so acquisition of these devices has become easier. At present, studies evaluating economic effects of telemonitoring in COPD are scant, with disparate results and large variability in methodology. The general trend, however, is that telemonitoring has the potential to produce cost reduction for the healthcare system $[2,3]$. Some studies even claim that the initial investment could pay off within the first year of the program implementation [23]. To our knowledge, there have been no previous reports on economic benefits of combined telemonitoring and home hospitalization in COPD.

In our study, patients showed a high degree of compliance with auto-monitoring program (data not shown), suggesting that use of communication technology is possible in the described population of patients. In addition, the patients demonstrated a high degree of satisfaction with the telemonitoring program, in agreement with previous observations [27]. Complaints related to interaction with technologies were few; however, in our study all patients had an available caregiver who could help them to use the devices correctly. Previous studies underline the importance of user-friendliness and ease of use of the technology for higher adherence levels and overall success of telemonitoring programs $[1,28]$.

The present study has several important limitations for the interpretation of the results. First, the number of participants was low, limited by the number of available devices and the restrictive selection criteria. Therefore, the results observed might have 
been affected/influenced by the patients not being included consecutively but rather selected according to predefined criteria, and the results may not be generalizable to a larger population. Second, this was a non-randomized study without a control group. Using historical data from the same patients as a control may be associated with recall bias, data incompleteness and distortions introduced by the natural evolution of the disease and changes in the health care system. Furthermore, during the control period (with no monitoring) the patients had access to the home hospitalization system following the standard circuits. It may well be that during the study period a greater effort was made to avoid hospital admission of patients, which could have partly contributed to the lower number of hospitalizations observed. This is a common limitation of non-randomized intervention studies. Third, the fact of participating in the study could influence the habits and adherence of the patients, which could introduce an additional bias in this before-and-after study.

In addition, during the study the patients were visited by the monitoring nurse, who provided recommendations on various health-related topics (inhalers, lifestyles), which did not occur in the control period and could also have potential positive effect on the observed results. Fourth, because of the time restrictions in the lending of telemonitoring devices, the follow-up period was limited to only six months. A longer follow-up period could have permitted to see a more pronounced effect of the intervention. Finally, the results of the satisfaction survey should be taken with caution, since the participants for this study were carefully selected and may not accurately represent the general population of patients with COPD. Moreover, the survey format limits the interpretation of the results, even when these are mostly satisfactory.

Modern technology allows for distant monitoring of various 610 clinical parameters and for exacerbation prediction through pattern analysis. However, technology should not replace care given by healthcare professionals, but serve as means to improve quality and optimize efficiency of care. There will always be a need for a team of nurses and physicians who would identify and interpret signals and intervene according to the therapeutic needs of the patient. Home hospitalization, which uses hospital-level resources and can provide complex care, can be an efficient and safe alternative to hospital admission and emergency room visits for many patients with decompensated chronic diseases, such as COPD.

\section{Conclusions}

Our observations suggest that combination of telemonitoring and home hospitalization may allow for reduction in the number of hospital admissions and visits to emergency room, compensated by an increased number of home hospitalizations. Such combined approach may help to reduce the economic burden of COPD exacerbations for the healthcare system.

\section{Key issues}

- Telemonitoring uses communication technology to monitor health status at distance. It is being used increasingly to remotely follow patients with chronic diseases, including
COPD. Telemonitoring allows for timely detection of alarm signals, upon which an action by health care professionals is required.

- Home hospitalization is a cost-saving alternative to inpatient hospitalization associated with increased patients' comfort and reduced risk of nosocomial infections. Nevertheless, the lack of constant proximity of and observation by the hospital staff may lead to a failure to detect early alarm signs.

- In this study, we evaluated the usefulness of a combined strategy based on telemonitoring and home hospitalization in reducing the number of hospital admissions and emergency room visits in patients with COPD.

- Twenty-eight patients with COPD (93\% males, mean age 78 years, mean baseline FEV1 50.8\%, 39\% using long-term oxygen therapy) monitored their health parameters at home using measuring devices connected to a web platform during 6 months.

- A specifically assigned nurse revised the collected parameters daily and performed programmed and on-demand phone calls and home visits. A home care unit (an internist and/or a geriatrist and/or nurses) was dispatched to the patients' home in case an alarm signal was detected. The outcomes were compared to historical data from the same patients.

- A total of 72 exacerbation episodes were registered in a total of $25(89.3 \%)$ patients during the study period (mean: 2.6 episodes per patient), versus 59 exacerbations in 26 (92.9\%) patients during the control period (mean: $2.1 \mathrm{epi}-$ sodes per patient; $p=0.21$ ).

- The number of conventional hospital admissions was reduced by $60 \%$ during the telemonitoring period (a total of 13 admission versus 33 admissions in the control period), whereas the number of visits to emergency room decreased by $38 \%$, from 53 visits during the control period to 33 visits during the telemonitoring period. Such reduction translated into higher number of home hospitalizations, which occurred on 57 occasions during the study period versus 12 occasions during the control period.

- Telemonitoring combined with home hospitalization was associated with reduced costs related to healthcare resource utilization (an estimated average saving of $1,860.8 €$ per patient per year).

- We conclude that such combined approach may help to reduce the costs related to disease management, however, in our experience it did not diminish the risk of COPD exacerbations.

\section{Author contributions}

Conception and design (MMR, RCF, IPP, API, CAL, SAV); Analysis and interpretation of the data (BMFF, IPP, MMR, RCF, JMM, EZL); Drafting the paper (MMR, IPP, BMFF, JGP, NMH, ADS); Revising the paper critically (SAV). All authors agree to be accountable for all aspects of the work.

\section{Funding}

This manuscript has received funding from the Torrejón public university hospital to fund editorial assistance provided by ContentEdNet . 


\section{Declaration of interest}

All technological support was provided by Cystelcom Sistemas S.A. (Madrid, Spain). The authors have no relevant affiliations or financial involvement with any organization or entity with a financial interest in or financial conflict with the subject matter or materials discussed in the manuscript. This includes employment, consultancies, honoraria, stock ownership or options, expert testimony, grants or patents received or pending, or royalties. Peer reviewers on this manuscript have no relevant financial or other relationships to disclose.

\section{References}

Papers of special note have been highlighted as either of interest ( $\bullet$ ) or of considerable interest (..) to readers.

1. Cruz J, Brooks D, Marques A. Home telemonitoring in COPD: a systematic review of methodologies and patients' adherence. Int J Med Inform. 2014;83(4):249-263.

2. Cruz J, Brooks D, Marques A. Home telemonitoring effectiveness in COPD: a systematic review. Int J Clin Pract. 2014;68(3):369-378.

3. McLean S, Nurmatov U, Liu JL, et al. Telehealthcare for chronic obstructive pulmonary disease. Cochrane Database Syst Rev. 2011 (7):CD007718

.- This systematic review of randomised clinical trials suggests that telemonitoring may have a positive impact on patients quality of life and on the number of admissions and emergency room visits.

4. Ekeland AG, Bowes A, Flottorp S. Effectiveness of telemedicine: a systematic review of reviews. Int J Med Inform. 2010;79(11):736771.

5. Pinnock H, Hanley J, McCloughan L, et al. Effectiveness of telemonitoring integrated into existing clinical services on hospital admission for exacerbation of chronic obstructive pulmonary disease: researcher blind, multicentre, randomised controlled trial. BMJ. 2013;347:f6070.

.. In this randomised controlled clinical trial telemonitoring did not increase neither time to admission nor health-related quality of life, as compared to usual care.

6. Ure J, Pinnock H, Hanley J, et al. Piloting tele-monitoring in COPD: a mixed methods exploration of issues in design and implementation. Prim Care Respir J. 2012;21(1):57-64.

- In this qualitative interview study patients reported that telemonitoring improved their access to healthcare professionals.

7. Mas MA, Santaeugenia S. [Hospital-at-home in older patients: a scoping review on opportunities of developing comprehensive geriatric assessment based services]. Rev Esp Geriatr Gerontol. 2015;50(1):26-34.

730 8. Sorknaes AD, Madsen H, Hallas J, et al. Nurse tele-consultations with discharged COPD patients reduce early readmissions-an interventional study. Clin Respir J. 2011;5(1):26-34.

9. Vitacca M, Bianchi L, Guerra A, et al. Tele-assistance in chronic respiratory failure patients: a randomised clinical trial. Eur Respir J. 2009;33(2):411-418.

10. Wong KW, Wong FKY, Chan MF. Effects of nurse-initiated telephone follow-up on self-efficacy among patients with chronic obstructive pulmonary disease. J Adv Nurs. 2005;49(2):210-222.

11. Aimonino Ricauda N, Tibaldi V, Leff B, et al. Substitutive "hospital at home" versus inpatient care for elderly patients with exacerbations of chronic obstructive pulmonary disease: a prospective randomized, controlled trial. J Am Geriatr Soc. 2008;56(3):493-500.

. In this study, telemonitoring was associated with a reduction in the number of admissions at 6 months, lower costs and improved quality of life in older patients with COPD exacerbations.
12. Puig-Junoy J, Casas A, Font-Planells J, et al. The impact of home hospitalization on healthcare costs of exacerbations in COPD patients. Eur J Health Econ. 2007;8(4):325-332.

- This study showed that home hospitalization of patients with COPD excarebations produced a mean saving of $36 \%$ in direct healthcare costs, compared with conventional hospitalization.

13. Mahoney FI, Barthel DW. Functional evaluation: the Barthel index. Md State Med J. 1965;14:61-65.

14. Shah S, Vanclay F, Cooper B. Improving the sensitivity of the Barthel index for stroke rehabilitation. J Clin Epidemiol. 1989;42 (8):703-709.

15. Pfeiffer E. A short portable mental status questionnaire for the assessment of organic brain deficit in elderly patients. J Am Geriatr Soc. 1975;23(10):433-441.

16. Charlson ME, Pompei $\mathrm{P}$, Ales $\mathrm{KL}$, et al. A new method of classifying prognostic comorbidity in longitudinal studies: development and validation. J Chronic Dis. 1987;40(5):373-383.

17. American Thoracic Society, medical section of the American Lung Association. Evaluation of impairment/disability secondary to respiratory disease. Am Rev Respir Dis. 1982;126(5):945-951.

18. Fletcher CM, Elmes PC, Fairbairn AS, et al. The significance of respiratory symptoms and the diagnosis of chronic bronchitis in a working population. Br Med J. 1959 29;2(5147):257-266.

19. Jones PW, Harding G, Berry $P$, et al. Development and first validation of the COPD assessment test. Eur Respir J. 2009;34 (3):648-654.

20. Diario oficial de Galicia. 2012 november 21;2012(222):43675.

21. Bourbeau J, Julien M, Maltais F, et al. Reduction of hospital utilization in patients with chronic obstructive pulmonary disease: a disease-specific self-management intervention. Arch Intern Med. 2003 10;163(5):585-591.

22. Pedone C, Chiurco D, Scarlata S, et al. Efficacy of multiparametric telemonitoring on respiratory outcomes in elderly people with COPD: a randomized controlled trial. BMC Health Serv Res. 2013;13:82.

23. De Toledo P, Jimenez S, Del Pozo F, et al. Telemedicine experience for chronic care in COPD. IEEE Trans Inf Technol Biomed. 2006;10 (3):567-573.

24. Polisena J, Tran K, Cimon K, et al. Home telehealth for chronic obstructive pulmonary disease: a systematic review and meta-analysis. J Telemed Telecare. 2010;16(3):120-127.

.. In this systematic review that included a total of $\mathbf{8 5 8}$ patients, home telemonitoring and telephone support were found to reduce rates of hospitalization and emergency department visits, although the mortality rate was greater in the telephone-support group compared with usual care.

25. Takahashi PY, Pecina JL, Upatising B, et al. A randomized controlled trial of telemonitoring in older adults with multiple health issues to prevent hospitalizations and emergency department visits. Arch Intern Med. 2012;172(10):773-779.

- In this randomized study with patients older than $\mathbf{6 0}$ years at high risk of rehospitalization, there were no differences niether in rates of admissions and emergency room visits nor in total length of hospital stay between telemonitoring and usual care groups.

26. Esteban C, Moraza J, Iriberri M, et al. Outcomes of a telemonitoringbased program (teIEPOC) in frequently hospitalized COPD patients. Int J Chron Obstruct Pulmon Dis. 2016;11:2919-2930.

27. Segrelles Calvo G, Gomez-Suarez C, Soriano JB, et al. A home telehealth program for patients with severe COPD: the PROMETE study. Respir Med. 2014;108(3):453-462.

28. Hoaas $\mathrm{H}$, Andreassen $\mathrm{HK}$, Lien $\mathrm{LA}$, et al. Adherence and factors affecting satisfaction in long-term telerehabilitation for patients with chronic obstructive pulmonary disease: a mixed methods study. BMC Med Inform Decis Mak. 2016;16:26. 